\title{
Network Analysis to Detect Common Strategies in the Italian Foreign Direct Investment
}

Giulia De Masi, Saipem (ENI)

Giorgia Giovannetti, Università degli Studi di Firenze e Fondazione M. Masi

Giorgio Ricchiuti, Università degli Studi di Firenze

First Draft: July 2009

Please do not quote without authors' permission

Comments are welcome

\begin{abstract}
In this paper, using the database ICE-Reprint, we study the network of Italian firms that invest abroad. Our analysis focuses on some manufacturing sectors, highlighting the linkages among firms and detecting the key nodes of the system (both in terms of firms and countries of destination). Moreover, through the examination of affiliates' economic activity we are able to distinguish different policies of internationalization among leaders.
\end{abstract}

Keywords: Foreign Direct Investment, Network Analysis 


\section{Introduction}

The recent and large literature on Globalization has highlighted as heterogeneous firms in developed countries have tackle in a heterogonous ways to the evolved international context: with quality upgrading of the products, employing migrants, splitting production in different countries, enhancing merger and acquisition of foreign firms and/or the establishment of subsidiaries abroad. Focusing on delocalization and exploiting the idea that the economic system is a natural network, we want to reconstruct and analyse the network of the Italian firms investing abroad. Studying at industrial level, different strategies of investments employed, to highlight the existence of heterogeneity.

Triggered by the opening up of the international markets, production networks have moved to a multi-country dimension. As a consequence, firms have modified their internationalization strategies making them more complex. As far as Italy is concerned, the empirical evidence at macroeconomic level reveals a low ability of domestic firms to penetrate foreign markets through FDI, especially during the last decade. This low multinational activity intensity of Italy is usually explained by the highly fragmented industrial structure, and by the small firms size, which makes it more difficult to fragment production and especially buy foreign firms (Mayer e Ottaviano, 2008; Mariotti e Mutinelli, 2006). Moreover, there is a strong heterogeneity among firms in terms of productivity, size and of the characteristics of the investments done abroad both regarding countries of destination and modes of internationalization. Modelling through network analysis these relations allows us to pull out information that usually does not outcrop: mainly we can understand whether the strategies of internationalization depend on proximity (at sector, and geographical level) among firms.disclosing the existence of differences between productive and commercial investments.

In the last decade, the analysis of complex networks has received great attention in both natural and social sciences (Dorogovtsev and Mendez 2003). Networks analysis enables the reconstruction of the linkages and the evolution of the connection between different individuals/agents/firms. Specifically, the main effort has been to understand the basic mechanism of communication networks: internet, World Wide Web, e-mails network (Vega-Redondo, 2007). Each of these systems is formed by a set of agents that interact and compete and can receive reciprocal advantages. This approach is promising for the study of economic systems where firms, households, individuals and the State act actively together, shaping without solution of continuity the relevant socio-economic structures. Network analysis is able to reproduce with simple model stylized facts, related to stationary and non-stationary contexts. Given improvements in computer 
science, these topics, initially analyzed in a game theoretic framework, have been recently developed using graph theory. Specifically, through networks the interactions are quantitatively analyzed by means of topological indexes. Pioneering empirical works in economics are related to the financial markets structure, the European firms' network and the relationship between firms and banks and flows of international trade (Caldarelli, 2006; Iori et al., 2006; Iori et al., 2007; De Benedicts e Tajoli, 2008).

We use the dataset ICE-Reprint (which holds data on Italian FDI) for 2005, achieving a bipartite graph where the nodes are investors and countries of destination. The topological structure depends on some characteristics: the same industrial sector, the same Italian province or doing investments in the same countries. Given the network dimension - there are 2934 investors, more that 137 countries of destination and 11000 affiliates - the analysis is limited to some particular manufacturing sectors (wearing, textile, electrical energy, machine and mechanical and electrical machineries) to understand:

1) whether the modes of internationalization depend on proximity (at sector, and geographical level);

2) what are the main hubs (countries/firms) within the sectors;

3) what are the strategies employed by the main actors (firms);

4) whether the main actors are the biggest firms.

This information is crucial to plan extensively an industrial policy. Indeed, the main concerns and critical remarks of the effects of globalization regard essentially the firm level, at which an active Government is required to safeguard both national production and employment within the country.

\section{The Dataset}

We employ the ICE-Reprint database, it is the census of foreign affiliates of Italian firms (with a turnover higher than 2.5 millions euros) in both manufactures and services sectors. However some financial sectors (i.e. banking, insurance, financial services) are not included. Moreover, the survey regards just equity (joint venture, participation with affiliates) but not non-equity operations. The 
Census has relevant information, regarding both Italian investors and affiliates: sales, number of employees, country of destination, industrial activity (for details, see Mariotti and Mutinelli, 2005).

For the division into industrial sectors we use the classification of economic activities ATECO (the Italian classification for NACE rev.2). This type of classification adopted by the Italian Institute of Statistics, is used for official statistical national economic analysis. We use the last version, which has been proposed in 2002.

In Table 1 we report some data regarding the whole dataset and the selected sectors. As already stated above, we consider 2934 investors from 94 Italian provinces and with 11023 affiliates distributed in 137 over 199 countries. In the manufacture sector on average, each investor has roughly four affiliates, with interesting differences among sectors considered in this paper: wearing, textile, energy and machine and mechanical and electrical machineries (therefore mechanical).

Table 1 Descriptive Statistics

\begin{tabular}{l|cccc} 
& Average Sales & $\begin{array}{c}\text { Average } \\
\text { Employees }\end{array}$ & $\begin{array}{c}\text { Number of } \\
\text { Affiliates }\end{array}$ & $\begin{array}{c}\text { Number of } \\
\text { Investors }\end{array}$ \\
\hline Wearing & $45.627(96.452)$ & $162(361)$ & 637 & 196 \\
Energy & $1655.091(3765.210)$ & $3076(8920)$ & 361 & 26 \\
Mechanical & $43.604(102.557)$ & $198(407)$ & 2156 & 617 \\
Textile & $36.464(52.043)$ & $179(248)$ & 635 & 245 \\
All Sectors & $610.656(1823.999)$ & $552(900)$ & 11023 & 2934
\end{tabular}

Note: Authors' calculation from ICE-Reprint. Standard Deviation in parenthesis.

It is worth noting that, on average, firms that invest abroad have more than 500 employees and more than 600 million euros of sales. However, there is a strong heterogeneity among sectors. Firms that produce Wearing and Textile are small and medium enterprises: just $10 \%$ of firms in these sectors employ more than 1000 workers. Moreover, the average sale is low in comparison with the average of the whole database: 36 and 45 millions of euros. On the other hand, the sector of energy 
(as well as those of chemical products and auto vehicles, non reported in Table 1) have a larger scale of dimension, both in terms of sales and of employees.

Looking at the geographical distribution of the affiliates, the three main markets of destination are USA, Germany and France, followed by Great Britain and Spain (Table 2). It is interesting that an evident second group is represented by new comers of EU (Romania, Poland and Hungary) and by Brazil and China. Particularly, in Romania delocalization is triggered by small and medium firms of both textile and mechanical sector.

Table 2 - Ten main countries of Destination

\begin{tabular}{l|cc} 
Country & $\begin{array}{c}\text { Number of } \\
\text { Investors }\end{array}$ & $\begin{array}{c}\text { Number of } \\
\text { Affiliates }\end{array}$ \\
\hline USA & 763 & 1196 \\
Germany & 710 & 1028 \\
France & 706 & 1201 \\
UK & 554 & 838 \\
Spain & 475 & 733 \\
Romania & 404 & 468 \\
Brazil & 287 & 363 \\
China & 273 & 375 \\
Polond & 226 & 270 \\
Hungary & 155 & 189
\end{tabular}

Note: Authors' calculation from ICE-Reprint.

\section{The Network of Italian FDI}

The intrinsic heterogeneity among firms leads to the adoption of different strategies of internationalization. The network analysis allows capturing and investigating those differences. As already stated, the nodes of the whole network are both Italian investors and host countries: it is a bipartite graph and a link is drawn if a particular investor goes in a particular host country (Peltomaki et al, 2006; Sneppen et al, 2004; Guillaume et al, 2004) . 
However, given the huge number of both nodes and linkages, we extract from the overall network, two sub-networks, each one composed by just one kind of nodes. Indeed, In the study of bipartite graph a very widely used approach is to separately study two networks that can be defined from the original network. If we call the two kinds of nodes as nodes A and B, we can study the network $G(A)+G(B)$ which has the total set of nodes $(A+B)$ or the networks $G(A)$ and $G(B)$ which have only nodes of kind A or B respectively (Strogatz, 2001; Newman, 2001). These two networks are called projected networks, in the sense that they are obtained as a projection of the initial graph in the subspace composed by nodes only of the same kind.

In our case, we can define the network of investors and the network of countries. The first is the network of Italian parent forms investing in the same country. The second one is the network of countries where the same firms invest. In Figure 1 and 2, we considered the projected networks of firms and countries respectively. To achieve a readable representation we considered just firms that have the highest number of links. Moreover, those (as all graphs below) are drawn using the Kamada-Kawai algorithm, particularly efficient to highlighting connections among groups, since, representing the graph as set of harmonic oscillators, where nodes are linked each other through springs, plotting in the middle of the figure those nodes (firms or countries of destination) with the highest number of edges and putting closely more connected nodes.

Studying Figure 1, the core of the projected network of firms is represented by the leaders of the Italian economic system. Wearing and Textile and Mechanical have firms well internationally integrated: on average they have more than one affiliate in 30 countries. However, analysing the activity of the affiliates (the kind of investment done by the investor), a great heterogeneity arise. Firstly there is a strong compresenza between FDI for production and those for commercial reason. The former are located in large and strategic countries used as launch pad while the latter are usually in other countries of the same continent. On the other hand, there are both firms that invest abroad through horizontal or vertical FDI and firms that invest in the most part of countries just through commercial activities or holdings (this interesting element may indicate the presence of local sub-contractors). Information related with different modes of internationalization is really useful to arrange an efficient industrial policy. Indeed, the effects on both employment and firm's performance strictly depend on the intensity of firms' international integration. 


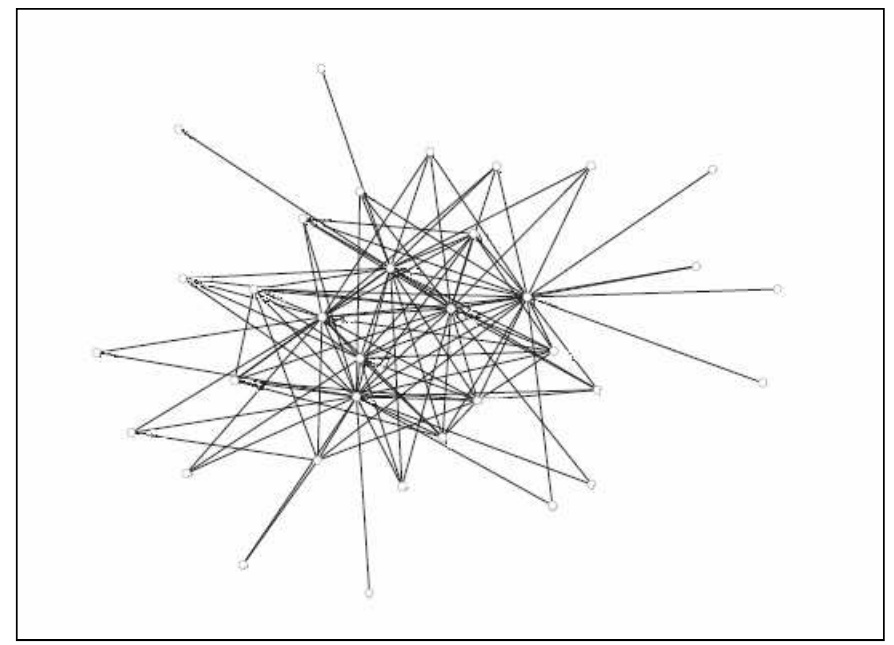

Figure 2 reports the projected network in the sub-space of countries. To detect the most important countries we highlighted a link between two countries if there are at least 500 investors in both countries. Given table 3, we reveal that the core of the network is composed by USA, France, UK, Spain and Germany: where USA is the highest hub but Germany is the node with the highest clustering coefficient. The connections around Germany are really dense (nodes linked with Germany are linked each other) meaning that the European country is a key country to understand the Italian FDI: it is really close, easily reachable even from small-medium enterprises. Other important markets are mainly countries of the European Union and Brazil, Argentina, China and India. This preliminary analysis allows us to detect both key markets of Italian firms and those markets for which Italy has still a gap in comparison with its competitors. 
Figure 2 Projected Network for country of destination: all sectors

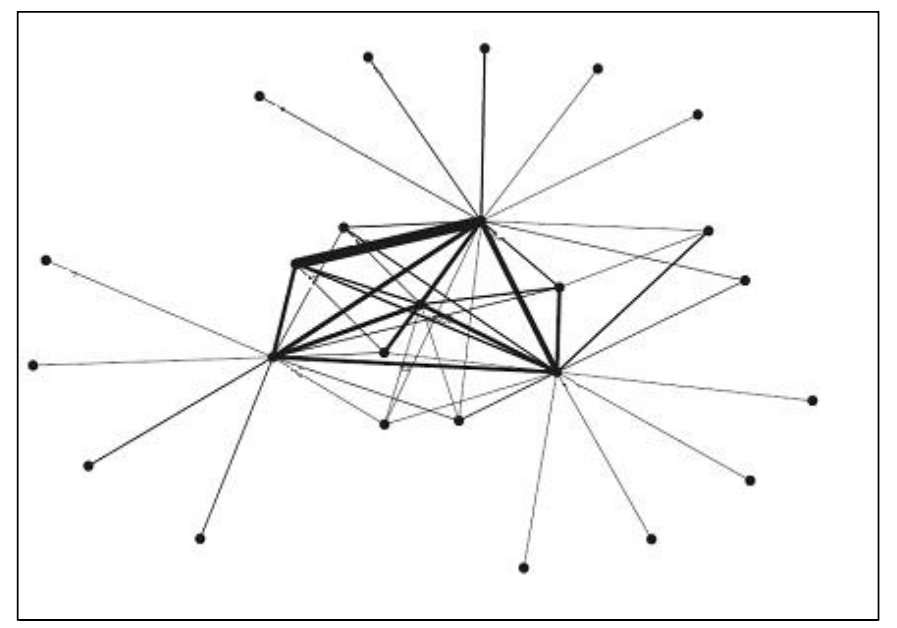

\section{Wearing and Textile}

Given nearness, we merged wearing and textile sectors. From a preliminary analysis it is worth noting the simultaneous presence of large and small-medium enterprises. (36\% of firms have less than 50 employees and 20\% less than15). The latter directs their investments predominantly towards countries of the European Union. Romania is an important attractor of these investments: roughly $50 \%$ of investors have less than 50 employees and half of these have less than 15 employees. Particularly, analysing the activity of the affiliates it seems that investments are done to reduce costs of production rather than to supply the neighbouring exporting markets. Table 3 reports some topological measures of the sector. The degree is 3.5: the lowest value among the sectors considered. The Closeness is high, indicating a strong density between nodes (firms). Finally the sector's betweeness (the average of the individual betweeness) is low: there are many key actors in this subspace. In general there are many common countries of destination and, as a consequence, homogeneity in the strategies adopted. 
Table 3 - Some topological measures

\begin{tabular}{l|rrrr} 
& $\begin{array}{c}\text { Number of } \\
\text { Investors }\end{array}$ & $\begin{array}{c}\text { Average } \\
\text { Degree }\end{array}$ & Closeness & Betweeness \\
\hline Wearing and Textile & 441 & 3.5 & 0.45 & 0.0007 \\
Mechanical & 617 & 4.0 & 0.46 & 0.0004 \\
Energy & 26 & 5.8 & 0.38 & 0.04
\end{tabular}

In table 4, we report correlations between degree and betweeness and some measure of size (sales, number of employees) for all sectors and for sectors considered. It is worth noting that for wearing ant textile the values are greater than those for all sectors: the larger the firms, the more numerous connected the firms are, the more relevant is their rule in the specific industrial network. This result seems to confirm, even if indirectly the analysis of Mayer and Ottaviano (2008), for who the more internationalized firms are the largest.

Table 4 - Correlation between topological measures and size

\begin{tabular}{l|cccc} 
Sector & (degree,sales) & $\begin{array}{l}\text { (degree, } \\
\text { employees) }\end{array}$ & $\begin{array}{l}\text { (betweeness,sales } \\
\text { (betweeness,employees) }\end{array}$ \\
\hline $\begin{array}{l}\text { Wearing and } \\
\text { Textile }\end{array}$ & 0.52 & 0.49 & 0.37 & 0.38 \\
Energy & 0.02 & 0.07 & 0.02 & 0.06 \\
Mechanical & 0.66 & 0.66 & 0.65 & 0.61 \\
All Sectors & 0.31 & 0.34 & 0.64 & 0.38
\end{tabular}

In Figure 3 and 4 we plotted the projected network for firms and countries of destination, respectively. In the network of firms there two clear elements: a key player and a group of firms (up in the right in the graph) which have similar - in terms of countries of destination - choices. Hence there exists a leader in the network, it has affiliates in the most important countries and it is trying to conquered new markets. Studying in detail the sector of its affiliates, it is worth noting that they are almost holding, maybe signalling the use of subcontracting as strategic policy. On the other hand the group of medium-large enterprises concentrates the presence in some specific markets (North America and West Europe). Moreover, their strategy of internationalization is unambiguous: they produce in one or at maximum two countries in the same subcontinent, at the same time they have 
one or more commercial offices in other countries of the same area. Their productive delocalization is, therefore, really definite and it is used as platform to export in the bordering countries. This result is crucial to evaluate the effects of FDI on the Italian economic system, because it seems that these firms do not invest abroad with cost-saving reasons but to enter in new markets (as bridgehead) to improve the performance of their exports.

Analaysing Figure 4, it is interesting that the fundamental nodes are again USA, France, Romania and Germany. In all these countries we have both commercial and productive activities. Moreover, there is a good presence of affiliates even in Japan and Hong Kong, with just commercial activities, and China with recent and growing productive activities.

Figure 3 Projected Network for Firms: Wearing and Textile

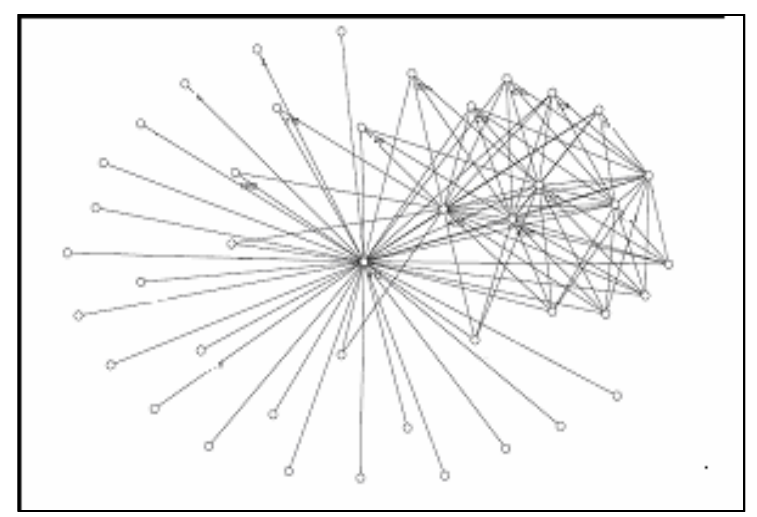

Note: to improve the visualization we removed both nodes and links with a lower weight (a lower number of countries of destination in common) 


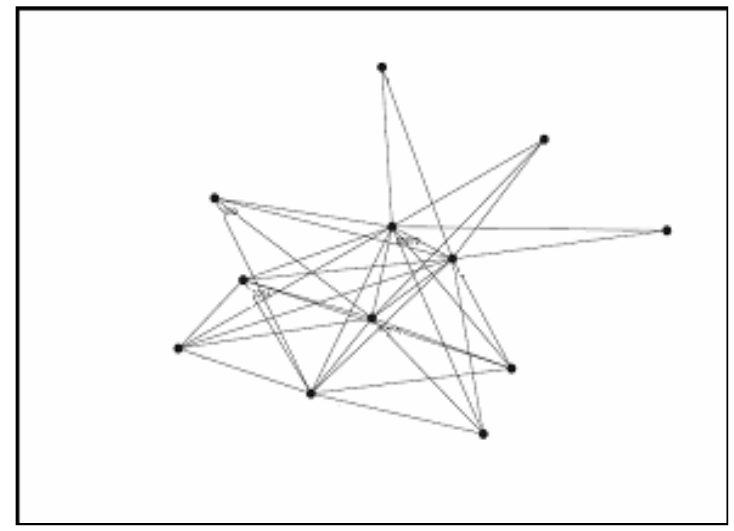

Note: to improve the visualization we removed both nodes and links with a lower weight (a lower number of countries of destination in common)

\section{Mechanical and electrical machineries (Mechanics)}

More than $20 \%$ of Italian manufacturing firms that invest abroad belong to the mechanical sector. They are small and medium enterprises: $75 \%$ have less than 250 employees and one fourth less than 50. On average a firm has 4 links and this value is strongly correlated with firms' size, both in terms of sales and employees. Firms in this sector invest in 83 countries with a strong presence in China (118) and Brazil (112) as well as farther countries such as Australia (38).

Figure 5 reports the projected network of investors. In the middle of the graph are easily evident three key nodes of the network: two firms which produce mechanical (apparecchi) and one that produce electrical machineries. All these firms produce just in two/three countries and have commercial activities all neighbouring countries: hence it seems a market seeking investment. These leaders are joined by other important players. Among these there are firms (all in the subsector of electrical machineries) which have done vertical investments mainly in developing or emerging countries such as China and Brazil.

The projected network of countries of destination (Fig. 6) shows two distinguish groups: leaders (the pentagon in the middle) and followers (Sweden, China, Brazil and the Nederland). Looking at the rule of affiliates in these countries, it is worth noting a relevant difference between these two sets. In the first group (with notice exception such as Germany and US), investors have 
just commercial activities. On the other hand in the second group there are mainly productive delocalization (both horizontal and vertical) and the commercial activity is just a by-product.

Figure 5 Projected Network for Firms: Mechanical

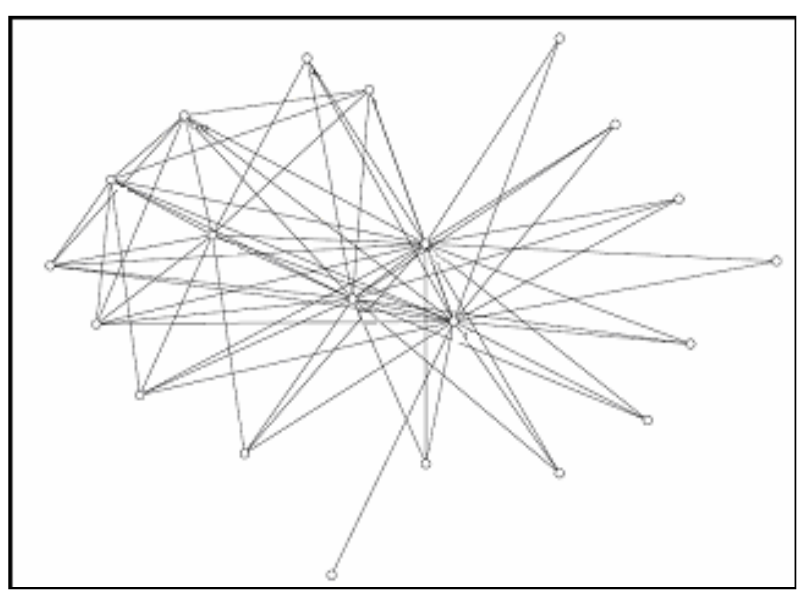

Note: to improve the visualization we removed both nodes and links with a lower weight (a lower number of countries of destination in common)

Figure 6 Projected Network for Countries of Destination: Mechanical

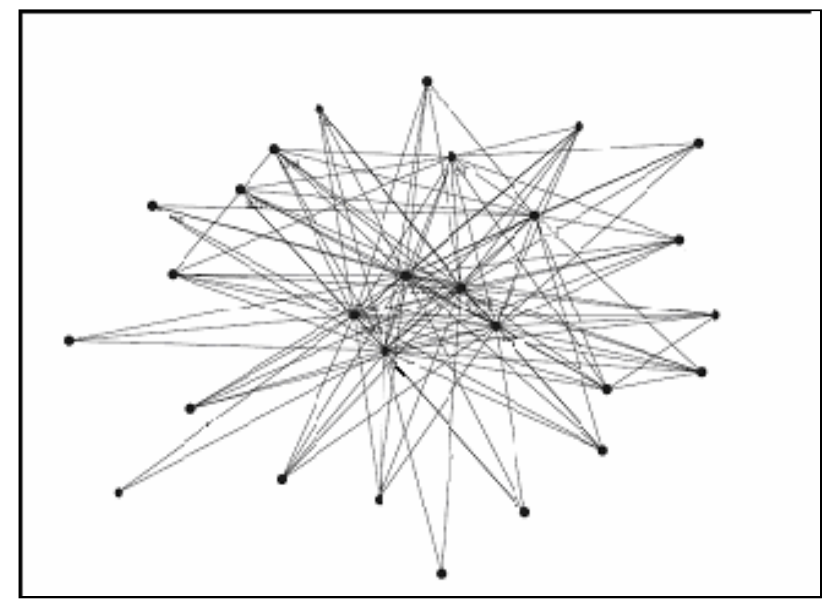

Note: to improve the visualization we removed both nodes and links with a lower weight (a lower number of countries of destination in common) 


\section{Electrical Energy (Energy)}

The sector of electrical energy presents, give the obvious effects of scale, the largest firms (both in terms of sales and employees, see Table 1), the lowest number of firms (26) and the largest number of average affiliates for investor (14). The average degree is 6 , detecting a greater dispersion of investments among countries of destination in comparison with the two sectors considered. The closeness is low while the betweeness is the biggest. This information suggests the presence of a dominant node (investor): differently from the other sectors where we found more big leaders. Indeed, $56 \%$ of affiliates belong to the same firm, linked to all the others, with significant presence in North and South America, and Spain. Its affiliates either produce and distribute energy or operate in sub-sector of services for electrical energy (mainly in US). However, there is even in this sector a second group of important players, whose affiliates mostly distribute energy. It is worth noting that while the leader is present in all continents, the activities of these firms are geographical concentrated in a key country (Argentina rather than Nederland). Finally, the key countries of destination for these sectors are those characterized by liberalization of both production and distribution of energy: mainly US and UK.

Figure 7 Projected Network for firms: Energy

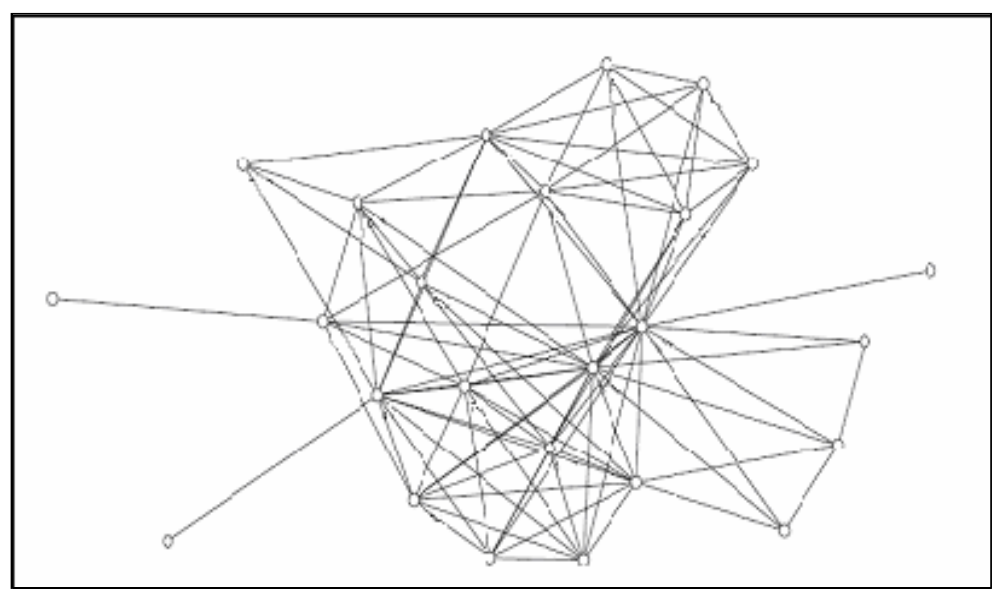

Nota: all investors 


\section{Figura 8 Projected Network for Countries of Destination: Energy}

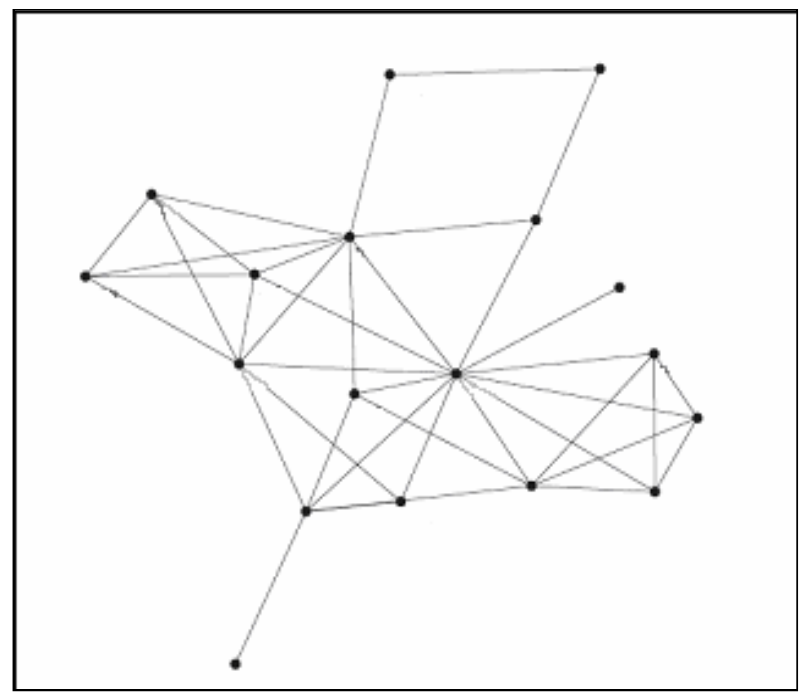

Note: to improve the visualization we removed both nodes and links with a lower weight (a lower number of countries of destination in common)

\section{Conclusions}

While some year ago trade was the most important mode of internationalization, nowadays firms are characterized by more complex strategies. However, in Italy the enterprises with a complete net of affiliates (for production or commercialization) are still few (and concentrated in some sectors). At the same time the internationalization has been associated with a strong reorganization of the production both within and between countries. Uncertainty linked with globalization has made aware politicians, businessmen and citizens that it is crucial monitoring constantly the evolution of the international markets, in order to analyse changes and minimizing the possible negative effects on the economic system specifically in terms of employment.

In this paper, using the database ICE-Reprint, we study the network of Italian firms that invest abroad. Our analysis focuses on some manufacturing sectors, highlighting the linkages among firms and detecting the key nodes of the system (both in terms of firms and countries of destination). Moreover, through the examination of affiliates' economic activity we are able to distinguish different policies of internationalization among leaders.

From our study emerges a strong heterogeneity (inter and intra-industries) of the strategies adopted. On the one hand there are firms that employ horizontal FDI using middle-large countries as productive pad to export in neighbouring countries through commercial affiliates. On the other hand, we have found global players, which make vertical FDI; their production is carried out for 
cost-saving reasons but also for professional qualities. Finally, the most part of foreign activities is done for commercial purposes, hence to promote Italian exports.

This work, detecting principal countries of destination (among the others US, France and Germany) and suggesting for which markets Italy is still late in comparisons with the other countries, could be exploited to support an active industrial policy for Italian internationalization. 


\section{Reference}

Caldarelli, G. (2006), Scale-Free Networks. Complex Webs in Nature and Technology, Oxford University Press.

De Benedictis L. e Tajoli L. (2008), La rete degli scambi commerciali mondiali, in ICE, Rapporto sul commercio estero - 2007/2008, Roma

Dorogovtsev, S.N. e J.F.F Mendez (2003), Evolution of networks, Oxford University Press.

Iori, G., G. De Masi, O. Precup, G. Gabbi e G. Caldarelli (2007), “A network analysis of the Italian overnight money market", in via di pubblicazione in Journal of Economic Dynamics and Control.

Iori, G., S. Jafarey e F. Padilla (2006), "Inter Bank Lending and Systemic Risk", in via di pubblicazione, Journal of Economic Behaviour and Organization.

Meyer T. e Ottaviano, G.M. (2008), The happy few: the internationalisation of European firms. New facts based on firm-level evidence, www.bruegel.org.

Mariotti, S. e Mutinelli, M. (2006), Italia Multinazionale 2006, Ed. Rubbettino, Roma

Vega-Redondo, F. (2007), Complex Social Networks, Econometric Society Monograph Series, Cambridge University Press. 